\title{
Separating Reflection Components based on Chromaticity and Noise Analysis
}

\author{
Robby T. Tan * Ko Nishino ${ }^{\dagger} \quad$ Katsushi Ikeuchi *
}

\begin{abstract}
Many algorithms in computer vision assume diffuse only reflections and deem specular reflections to be outliers. However, in the real world, the presence of specular reflections is inevitable, since there are many dielectric inhomogeneous objects which have both diffuse and specular reflections. To resolve this problem, we present a method to separate the two reflection components. The method is principally based on the distribution of specular and diffuse points in a two-dimensional maximum chromaticity-intensity space. We found that, by utilizing the space and known illumination color, the problem of reflection component separation can be simplified into the problem of identifying diffuse maximum chromaticity. To be able to identify the diffuse maximum chromaticity correctly, an analysis of the noise is required, since most real images suffer from it. Unlike existing methods, the proposed method can separate the reflection components robustly for any kind of surface roughness and light direction.
\end{abstract}

Index Terms: Reflection components separation, specular reflection, diffuse reflection, dichromatic reflection model, noise analysis, chromaticity, specular-to-diffuse mechanism.

\section{Introduction}

Highlights reflected from inhomogeneous objects are linear combinations of diffuse and specular reflection components. The presence of highlights causes many algorithms in computer vision to produce erroneous results; since, most of the algorithms assume diffuse only reflections and deem specular reflections as outliers. To properly acquire the diffuse only reflections, a method to separate

\footnotetext{
${ }^{1}$ This research was, in part, supported by Japan Science and Technology (JST) under CREST Ikeuchi Project.

*Department of Computer Science, The University of Tokyo, Japan, E-mail: \{robby,ki\} @cvl.iis.u-tokyo.ac.jp

${ }^{\dagger}$ Department of Science, Columbia University, USA, E-mail: kon@cs.columbia.edu
} 
the two components robustly and accurately is required. Once this separation has been accomplished, knowledge of the specular reflection component becomes advantageous, since it conveys useful information about surface properties such as microscopic roughness.

Many methods have been developed to separate reflection components. Wolff et al. [15] and Nayar et al. [11] used a polarizing filter to identify pixels that had a specular reflection component. Generally, methods using polarizing filters are sufficiently accurate to separate reflection components; however, using such additional devices is impractical in some circumstances. Sato et al. [12] introduced a four-dimensional space - temporal-color space - to analyze the diffuse and specular reflections based solely on color. While their method has the ability to separate the reflection components locally, it requires dense input images with a variation of illuminant directions. Recently, instead of using dense images, Lin et al. [10] used sparse images under different illumination positions to resolve the separation problem. They used an analytical method that combines the finite dimensional basis model and dichromatic model to form a closed form equation by assuming that the sensor sensitivity is narrowband. Other methods using multiple images can be found in the literature $[7,9]$.

Shafer [13], who proposed the dichromatic reflection model, was one of the early researchers who used a single uniformly colored image. He proposed a separation method based on parallelogram distribution of colors in RGB space. Klinker et al. [4] then extended this method by introducing a T-shaped color distribution, which was composed of reflectance and illumination color vectors. Unfortunately, for most real images, this T shape is rarely extractable due to noise, etc. Bajscy et al. [1] proposed a different approach that introduced a three dimensional space composed of lightness, saturation and hue. In their method, the input image whose illumination color is known has to be neutralized to pure-white illumination using a linear basis functions operation. Although this method is more accurate than the method of Klinker et al. [4], it requires correct specular-diffuse pixel segmentation, which is dependent on camera parameters.

In this paper, we describe our goal: to separate the reflection components of uniformly colored surfaces from a single input image. To accomplish this, we base the method on chromaticity, particularly on the distribution of specular and diffuse points in maximum chromaticity-intensity space. Briefly, the method is as follows. Given a single colored image taken under a uniformly colored illumination, we first identify the diffuse pixel candidates based on color ratio and noise analysis, particularly camera noise. We normalize both input image and diffuse pixel candidates simply by dividing their pixels values with known illumination chromaticity. Color constancy algorithms (e.g., $[14,2])$ can be employed to estimate the illumination chromaticity. From the normalized diffuse candidates, we estimate the diffuse maximum chromaticity by using histogram analysis. Having obtained the normalized image and the normalized diffuse maximum chromaticity, the separation can be done straightforwardly using a specular-to-diffuse mechanism, a new mechanism which we introduce. 
Finally, we renormalize the reflection components to obtain the actual reflection components.

Although the method can be used to deal with multicolored surfaces by employing color segmentation (e.g., region growing algorithms [8] based on hue), in this paper we focus the discussion on uniformly colored surfaces, since we intend to underline the robustness and the accuracy of the method. In our experiment the separation results will be evaluated by comparison with the results of using polarizing filters. Considering the advantages of the method, it should be useful for color-based object recognition, color indexing, or other computer vision algorithms as well. Such algorithms usually require a consistent color descriptor of an object; however, highlights could cause the color appearance of the object to be inconsistent with respect to the change of viewing and illumination direction. Thus, by successfully acquiring diffuse only reflection, we become able to provide a consistent color descriptor of an object. Note that, in this paper, we also introduce a novel two-dimensional space: maximum chromaticity-intensity space. Previously, a number of researchers (e.g., [5]) have utilized chromaticity space for color analysis; however, since the differences of specular and diffuse reflections are not only due to color but also to intensity, we combine both of them in a single space.

The rest of the paper is organized as follows. In Section 2, we discuss the dichromatic model of inhomogeneous materials and image color formation. In Section 3, we explain the method in detail, describing the derivation of the theory and the algorithm for separating diffuse and specular reflection components. We provide a brief description of the implementation of the method and experimental results for real images in Section 4. Finally in Section 5, we offer our conclusions.

\section{Reflection Models}

Based on the dichromatic reflection model [13] and image formation of a digital camera, we can describe camera response or image intensity as:

$$
\mathbf{I}(\mathbf{x})=w_{d}(\mathbf{x}) \int_{\Omega} S(\lambda) E(\lambda) \mathbf{q}(\lambda) d \lambda+w_{s}(\mathbf{x}) \int_{\Omega} E(\lambda) \mathbf{q}(\lambda) d \lambda
$$

where $\mathbf{I}=\left\{I_{r}, I_{g}, I_{b}\right\}$, the color vector of camera response or image intensity; $\mathbf{x}=\{x, y\}$, the two dimensional image coordinates, and $\mathbf{q}=\left\{q_{r}, q_{g}, q_{b}\right\}$ is the three-element-vector of sensor sensitivity. $w_{d}(\mathbf{x})$ and $w_{s}(\mathbf{x})$ are the weighting factors for diffuse and specular reflection, respectively; their values depend on the geometric structure at location $\mathbf{x} . S(\lambda)$ is the diffuse spectral reflectance function, while $E(\lambda)$ is the spectral energy distribution function of illumination. These two spectral functions are independent of the spatial location $(\mathbf{x})$ because we assume a uniform surface color as well as a uniform illumination color. The integration is done over the visible spectrum $(\Omega)$. Note that we ignore the camera gain and camera noise in the above model, and assume that the model follows the neutral interface reflection (NIR) assumption [6], i.e., the color of specular reflection component equals the 
color of the illumination. For the sake of simplicity, Equation (1) can be written as:

$$
\mathbf{I}(\mathbf{x})=w_{d}(\mathbf{x}) \mathbf{B}+w_{s}(\mathbf{x}) \mathbf{G}
$$

where $\mathbf{B}=\int_{\Omega} S_{d}(\lambda) E(\lambda) \mathbf{q}(\lambda) d \lambda$, and $\mathbf{G}=\int_{\Omega} E(\lambda) \mathbf{q}(\lambda) d \lambda$. The first part of the right side of the equation represents the diffuse reflection component, while the second part represents the specular reflection component.

Chromaticity We define chromaticity or normalized rgb as:

$$
\boldsymbol{\sigma}(\mathbf{x})=\frac{\mathbf{I}(\mathbf{x})}{I_{r}(\mathbf{x})+I_{g}(\mathbf{x})+I_{b}(\mathbf{x})}
$$

where $\boldsymbol{\sigma}=\left\{\sigma_{r}, \sigma_{g}, \sigma_{b}\right\}$. Based on the equation, for diffuse only reflection component $\left(w_{s}=0\right)$, the chromaticity will be independent from the diffuse geometrical parameter $w_{d}$. We call this diffuse chromaticity $(\boldsymbol{\Lambda})$ with definition:

$$
\mathbf{\Lambda}=\frac{\mathbf{B}}{B_{r}+B_{g}+B_{b}}
$$

where $\boldsymbol{\Lambda}=\left\{\Lambda_{r}, \Lambda_{g}, \Lambda_{b}\right\}$ and $\mathbf{B}=\left\{B_{r}, B_{g}, B_{b}\right\}$. On the other hand, for specular only reflection component $\left(w_{d}=0\right)$, the chromaticity will be independent from the specular geometrical parameter $\left(w_{s}\right)$, and we call it specular chromaticity or illumination chromaticity $(\boldsymbol{\Gamma})$ :

$$
\Gamma=\frac{\mathbf{G}}{G_{r}+G_{g}+G_{b}}
$$

where $\boldsymbol{\Gamma}=\left\{\Gamma_{r}, \Gamma_{g}, \Gamma_{b}\right\}$ and $\mathbf{G}=\left\{G_{r}, G_{g}, G_{b}\right\}$. Consequently, regarding to Equation (4) and (5), Equation (2) becomes able to be written in term of chromaticity:

$$
\mathbf{I}(\mathbf{x})=m_{d}(\mathbf{x}) \boldsymbol{\Lambda}+m_{s}(\mathbf{x}) \mathbf{\Gamma}
$$

where

$$
\begin{aligned}
& m_{d}(\mathbf{x})=w_{d}(\mathbf{x})\left(B_{r}+B_{g}+B_{b}\right) \\
& m_{s}(\mathbf{x})=w_{s}(\mathbf{x})\left(G_{r}+G_{g}+G_{b}\right)
\end{aligned}
$$

As a result, we have three types of chromaticity: image chromaticity $(\boldsymbol{\sigma})$, diffuse chromaticity $(\Lambda)$ and illumination chromaticity $(\Gamma)$. The image chromaticity is directly obtained from the input image using Equation (3). In addition, we can obtain that $\left(\sigma_{r}+\sigma_{g}+\sigma_{b}\right)=\left(\Lambda_{r}+\Lambda_{g}+\Lambda_{b}\right)=\left(\Gamma_{r}+\Gamma_{g}+\Gamma_{b}\right)=1$.

Problem Definition and Constraints Given image intensities (I) whose illumination chromaticity $(\boldsymbol{\Gamma})$ is estimated using existing color constancy method, we intend to decompose them into their reflection components: $m_{d}(\mathbf{x}) \boldsymbol{\Lambda}$ and $m_{s}(\mathbf{x}) \boldsymbol{\Gamma}$, described in Equation (6). To accomplish the decomposition or separation correctly, our proposed method requires two constraints: first, the surface chromaticity should be chromatic (the distribution of $S(\lambda)$ in Equation (1) is not flat); and second, the camera responses are linear to the flux of incident intensities. 


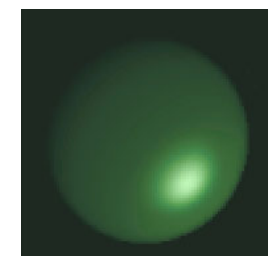

(a)

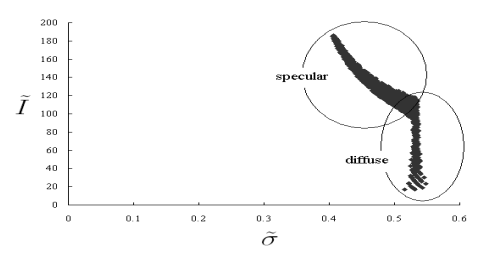

(b)

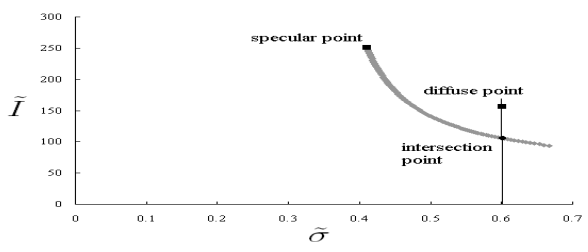

(c)

Figure 1: (a) Synthetic image (b) the projection of the synthetic image pixels into the maximum chromaticity intensity space (c) specular point distribution when the value of $m_{s}$ changes continually and the value of $m_{d}$ is constant.

\section{Separation Method}

In this section, we first deal with uniformly colored surfaces that have a pure-white specular component $\left(\Gamma_{r}=\Gamma_{g}=\Gamma_{b}\right)$. Then, we encounter more realistic images where $\Gamma_{r} \neq \Gamma_{g} \neq \Gamma_{b}$ by utilizing a normalization technique.

\subsection{Specular-to-diffuse mechanism}

To separate the reflection components, we basically rely on a specular-to-diffuse mechanism. This mechanism is based on maximum chromaticity and intensity values of diffuse and specular pixels. Following the chromaticity definition in Equation (3) we define maximum chromaticity as:

$$
\tilde{\sigma}(\mathbf{x})=\frac{\tilde{I}(\mathbf{x})}{I_{r}(\mathbf{x})+I_{g}(\mathbf{x})+I_{b}(\mathbf{x})}
$$

where $\tilde{I}(\mathbf{x})=\max \left(I_{r}(\mathbf{x}), I_{g}(\mathbf{x}), I_{b}(\mathbf{x})\right)$. Unlike chromaticity $(\boldsymbol{\sigma})$ which is a color vector, maximum chromaticity $(\tilde{\sigma})$ is a scalar value. By assuming a uniformly colored surface lit with a single colored illumination, in a two-dimensional space: maximum chromaticity-intensity space, where $x$-axis representing $\tilde{\sigma}$ and $y$-axis representing $\tilde{I}$, the maximum chromaticities of diffuse points (points whose $m_{d}>0$ and $m_{s}=0$ ) will be always larger than those of specular points (points whose $m_{d}>0$ and $m_{s}>0$ ) due to the maximum operator in the definition of $\tilde{I}$ and due to the assumption that the illumination is achromatic. Moreover, based on Equation (9), the maximum chromaticities of diffuse points will be constant regardless of the variance of $m_{d}$. In contrast, the maximum chromaticities of specular points will vary with regard to the variance of $m_{s}$, as shown in Figure 1.b. From these different characteristics of specular and diffuse points in the maximum chromaticity intensity space, we devised the specular-to-diffuse mechanism. The details are as follows. When two pixels, a specular pixel $\mathbf{I}\left(\mathbf{x}_{1}\right)$ and a diffuse pixel $\mathbf{I}\left(\mathbf{x}_{2}\right)$, with exactly the same diffuse chromaticity $(\Lambda)$ are projected into maximum chromaticity-intensity space, the maximum chromaticity $(\tilde{\sigma})$ of the diffuse point will be larger than that of the specular point. If the color of the specular component is pure white: $\Gamma_{r}\left(\mathbf{x}_{\mathbf{1}}\right)=\Gamma_{g}\left(\mathbf{x}_{\mathbf{1}}\right)=\Gamma_{b}\left(\mathbf{x}_{\mathbf{1}}\right)$, by subtracting all color channels of the specular pixel's intensity 
with a small scalar number iteratively, and then projecting those subtracted image intensities into the space, we will find that the points form a curved line, as shown in Figure 1.c. This curved line follows the below Equation: (see the Appendix for complete derivation):

$$
\tilde{I}(\mathbf{x})=m_{d}(\mathbf{x})(\tilde{\Lambda}-1 / 3)\left(\frac{\tilde{\sigma}(\mathbf{x})}{\tilde{\sigma}(\mathbf{x})-1 / 3}\right)
$$

where $\tilde{\Lambda}$ is the $\Lambda_{c}$ of $\tilde{I}$, with index $c$ is identical to the color channel of $\tilde{I}$. Note that this equation also proves that the distribution of specular points in maximum chromaticity intensity space forms a curved cluster if the values of $m_{d}$ vary (Figure 1.b).

In Figure 1.c, we can observe that a certain point in the curved line intersects with a vertical line representing the maximum chromaticity of the diffuse point. At this intersection, $m_{s}$ of the specular pixel equals zero, since the maximum chromaticity of the subtracted specular pixel becomes identical to that of the diffuse pixel. As a consequence, the intersection point becomes crucial (the point indicates the diffuse component of the specular pixel). Mathematically, to obtain the diffuse component, we first compute $m_{d}\left(\mathbf{x}_{1}\right)$, which can be derived from Equation (10):

$$
m_{d}\left(\mathbf{x}_{\mathbf{1}}\right)=\frac{\tilde{I}\left(\mathbf{x}_{\mathbf{1}}\right)\left[3 \tilde{\sigma}\left(\mathbf{x}_{\mathbf{1}}\right)-1\right]}{\tilde{\sigma}\left(\mathbf{x}_{\mathbf{1}}\right)\left[3 \tilde{\Lambda}\left(\mathbf{x}_{\mathbf{1}}\right)-1\right]}
$$

To compute the value of $m_{d}$ we need to know the value of $\tilde{\Lambda}$. This value can be obtained from the diffuse pixel, since if the two pixels have the same diffuse chromaticity, then $\tilde{\Lambda}\left(\mathbf{x}_{1}\right)=\tilde{\Lambda}\left(\mathbf{x}_{\mathbf{2}}\right)=\tilde{\sigma}\left(\mathbf{x}_{\mathbf{2}}\right)$. Having known the value of $m_{d}\left(\mathbf{x}_{1}\right)$, we can directly obtain the value of $m_{s}\left(\mathbf{x}_{1}\right)$, since:

$$
m_{s}\left(\mathbf{x}_{\mathbf{1}}\right)=\left(I_{r}\left(\mathbf{x}_{\mathbf{1}}\right)+I_{b}\left(\mathbf{x}_{\mathbf{1}}\right)+I_{g}\left(\mathbf{x}_{\mathbf{1}}\right)\right)-m_{d}\left(\mathbf{x}_{\mathbf{1}}\right)
$$

Finally, the diffuse reflection component of the specular pixel can be computed as: $m_{d}\left(\mathbf{x}_{\mathbf{1}}\right) \boldsymbol{\Lambda}=$ $\mathbf{I}\left(\mathbf{x}_{\mathbf{1}}\right)-m_{s}\left(\mathbf{x}_{\mathbf{1}}\right) \frac{1}{3}$.

Based on the above mechanism, therefore, the problem of reflection components separation can be simplified into the problem of finding diffuse maximum chromaticity ( $\tilde{\sigma}$ of diffuse pixels). For synthetic images, which have no noise, the diffuse maximum chromaticities are constant and thus trivial to find. Figure 1.b, shows the distribution of synthetic image pixels in maximum chromaticity-intensity space. By considering Equation (9), we can obtain the diffuse maximum chromaticity from the largest maximum chromaticity value (the extreme right of the point cloud). Then, with regard to this diffuse maximum chromaticity, we can accomplish the separation pixel by pixel in a straightforward manner using the above mechanism. Figure $2 . b \sim c$ show the separation result. For real images, unfortunately, instead of forming constant values, the diffuse maximum chromaticities vary within a considerably wide range (Figure 2.e). This is due to imaging noises and surface non-uniformity (although human perception perceives a uniform color, in fact in the real world, there is still surface non-uniformity due to dust, imperfect painting, etc.). Therefore, to correctly and robustly obtain the diffuse maximum chromaticity, we should include those noises in our analysis. Note that the specular-to-diffuse 


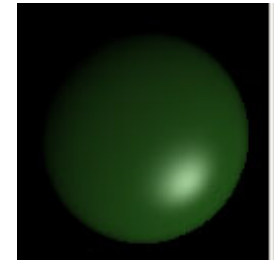

(a)

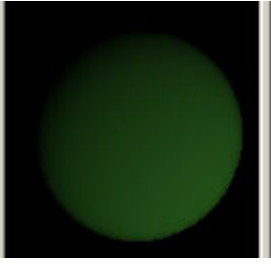

(b)

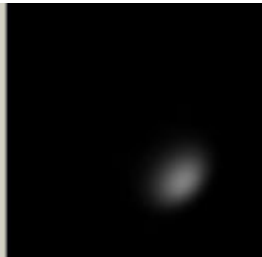

(c)

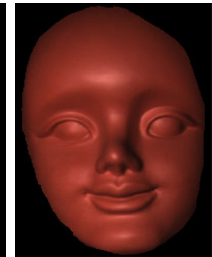

(d)

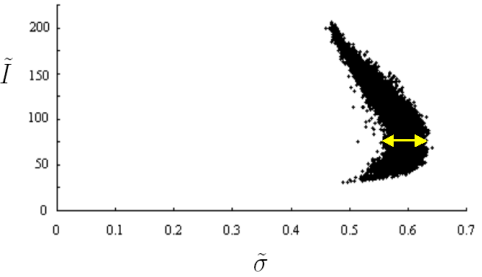

(e)

Figure 2: (a) Synthetic input image (b) diffuse component (c) specular component (d) real input image (e) the projection of the real image into maximum chromaticity-intensity space

mechanism requires linearity between the camera output and the flux of incoming light intensity, and works only on chromatic surfaces.

\subsection{Color Ratio and Noise Analysis}

In this section, first we intend to show that in real images, noise can make the values of diffuse chromaticity $(\Lambda)$ of the image vary, although human perception deems that the images contain uniformly colored surfaces (constant $\Lambda$ ). Second, from the varied values of $\Lambda$, we will group the points that have the same $\Lambda$, which ideally means points that have exactly the same surface color and noise. We expect that by transforming each of these groups into maximum chromaticity-intensity space, we can obtain a single diffuse maximum chromaticity as in synthetic images.

Color Ratio To accomplish the two tasks above we use color ratio, which we define as:

$$
u=\frac{I_{r}+I_{b}-2 I_{g}}{I_{g}+I_{b}-2 I_{r}}
$$

where $u$ is a scalar value. The location parameter $(\mathbf{x})$ is removed, since we work on each pixel independently. For a pure-white specular reflection component $\left(\Gamma_{r}=\Gamma_{g}=\Gamma_{b}\right)$, the color ratio $(u)$ for both diffuse and specular pixels can be described as a function of $\Lambda$ alone:

$$
u=\frac{\Lambda_{r}+\Lambda_{b}-2 \Lambda_{g}}{\Lambda_{g}+\Lambda_{b}-2 \Lambda_{r}}
$$

The last equation means that, if two pixels have the same diffuse chromaticity $(\Lambda)$, they will have the identical value of $u$ regardless of whether they are diffuse or specular pixels (specularity independence). In addition, the value of $u$ is also independent from shading and shadows; although, the independence from shadow is fulfilled if the ambient illumination has the same spectral energy distribution to the direct illumination.

Based on Equation (13), we create a two-dimensional space: $u$-intensity space, with $u$ as $x$-axis and $\tilde{I}$ as $y$-axis. By projecting all pixels of a real image into this space, we obtain a cloud of points as shown in Figure 3.a. Ideally, if the surface color is perfectly unique and there is no noise from the 


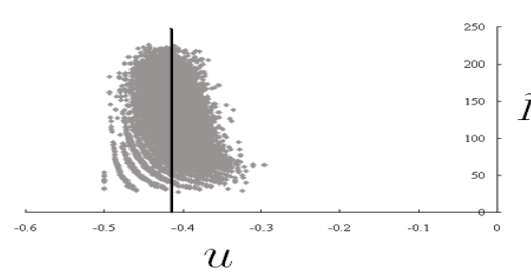

(a)

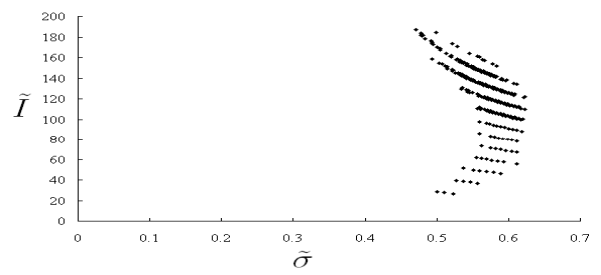

(b)

Figure 3: (a) The projection of the pixels of Figure 2.d into $u$-intensity space (b) the projection of pixels that correspond to points crossed by a vertical line in the left figure.

camera, we should observe only a single straight line in this space since the values of $u$ are constant. However, as can be seen in the figure, this does not hold true for real images. This is mainly due to the slight variation of surface color and illumination color as well as to noise produced through the camera sensing process, which are imperceptible to human eyes. In our analysis, we assume that the variance of illumination color is small, and neglect it. Thus, the variance of the color ratio in the space is caused by surface non-uniformity and camera noise.

According to Equation (14), the varying values of $u$ implies the varying values of $\Lambda$. However, as shown in Figure 3.a, not all values of $u$ are completely varied, there are still a number of groups of points that have the same value of $u$. If we select one of these groups, we can obtain a number of pixels that most likely have identical $\Lambda$. For these pixels, we can expect that, by transforming them into maximum chromaticity-intensity space, their distribution can be the same as the distribution of synthetic image which is free from noise (since we have ensured that the values $\Lambda$ are constant). If this expectation holds true, then the diffuse maximum chromaticity can be obtained in a straightforward manner. Figure 3.a shows a vertical line at a certain value of $u$. Pixels that correspond to the points crossed by the vertical line have identical values of $u$. Figure 3.b shows the transformation of the pixels into maximum chromaticity-intensity space. While the distribution of specular points forming curved lines fits our explanation (Equation (10)), the distribution of diffuse points is beyond our expectation since, instead of forming a single vertical line, they form a number of curved lines. Consequently, we cannot straightforwardly obtain the diffuse maximum chromaticity. Moreover, the curved distribution of diffuse points also indicates that the dichromatic reflection model alone is insufficient to explain the behavior of diffuse points in maximum chromaticity-intensity space. We require a model that covers camera noise.

Camera Noise Model We model camera noise for a diffuse pixel as:

$$
\mathbf{I}(\mathbf{x})=m_{d}(\mathbf{x}) \boldsymbol{\Lambda} \boldsymbol{\theta}(\mathbf{x})+\boldsymbol{\phi}(\mathbf{x})
$$

where $\boldsymbol{\theta}=\left\{\theta_{r}, \theta_{g}, \theta_{b}\right\}$ and $\boldsymbol{\phi}=\left\{\phi_{r}, \phi_{g}, \phi_{b}\right\}$ are the first and second types of camera noise in the three sensor channels, respectively. The two types of camera noise depend on the spatial location $(\mathrm{x})$ 
indicating that the noise varies throughout the image. The above model is a simplification of a more complex model proposed by Healey et al. [3]. According to the model, there are two types of camera noise, namely, noise that is dependent on incoming intensity (shot noise), and noise that is independent of incoming intensity (dark noise). In our simplified model, $\boldsymbol{\theta}$ is identical to the intensity-dependent noise, implying $\theta_{r}(\mathbf{x}) \neq \theta_{g}(\mathbf{x}) \neq \theta_{b}(\mathbf{x})$. While, $\boldsymbol{\phi}$ is identical to the intensity-independent noise, implying $\phi_{r}(\mathbf{x})=\phi_{g}(\mathbf{x})=\phi_{b}(\mathbf{x})$, regardless of the color of incident light. Based on the noise model in Equation (15), we can consider that the variance of $u$ in Figure 3.a originates from non-constant values of $\boldsymbol{\Lambda}$ and $\boldsymbol{\theta}$.

By plugging Equation (15) into Equation (13), $u$ for diffuse pixels becomes:

$$
u=\frac{m_{d}\left(\Lambda_{r} \theta_{r}+\Lambda_{b} \theta_{b}-2 \Lambda_{g} \theta_{g}\right)+\left(\phi_{r}+\phi_{b}-2 \phi_{g}\right)}{m_{d}\left(\Lambda_{g} \theta_{g}+\Lambda_{b} \theta_{b}-2 \Lambda_{r} \theta_{r}\right)+\left(\phi_{g}+\phi_{b}-2 \phi_{r}\right)}
$$

Based on the last equation, if we have two diffuse pixels, their $u$ values will be the same if their combination of $\boldsymbol{\Lambda}$ and $\boldsymbol{\theta}$ are identical, since $\phi_{r}(\mathbf{x})=\phi_{g}(\mathbf{x})=\phi_{b}(\mathbf{x})$. However, for diffuse pixels with noise, the same value of $u$ does not always imply the same value of maximum chromaticity $(\tilde{\sigma})$. Since, if their second noise values are different $\left[\phi\left(\mathbf{x}_{1}\right) \neq \phi\left(\mathbf{x}_{2}\right)\right]$, then the values of maximum chromaticity $(\tilde{\sigma})$ are also different. This condition is the cause of curved lines distribution of diffuse points in maximum chromaticity-intensity space; namely, the second type of noise in diffuse pixels behaves like the specular reflection component $\left(\phi\right.$, where $\phi_{r}=\phi_{g}=\phi_{b}$, behaves like $m_{s}$, a scalar value). Moreover, like specular points, the number of curved lines in the space is also determined by the number of different $m_{d}$. The range of diffuse maximum chromaticities depends on camera noise characteristics which could be different from camera to camera. Importantly, since $\phi$ behaves like $m_{s}$, the diffuse curved distribution can be detected by using Equation (10). This detection is crucial since we intend to find the actual diffuse maximum chromaticity.

Having characterized the diffuse distribution and identified each of them by utilizing Equation (10), we can determine the actual diffuse pixels (diffuse pixels that do not suffer from the second type of noise) by choosing a certain point in every diffuse curved line. By assuming that $\phi$ contains identical positive numbers, then the actual diffuse pixels are pixels that have $\phi=\{0,0,0\}$. Consequently, the actual diffuse pixels correspond to diffuse points that have the smallest intensity (the points that have the biggest chromaticity in each curved line). However, since several curved lines might have no point whose $\phi=\{0,0,0\}$, we cannot claim that all smallest points in the curved lines correspond to the actual diffuse pixels; thus, we call the points diffuse point candidates and their corresponding pixels diffuse pixel candidates.

Besides affecting diffuse pixels, the two types of noise also affect specular pixels, which can be described as $\mathbf{I}(\mathbf{x})=m_{d}(\mathbf{x}) \boldsymbol{\theta}(\mathbf{x}) \boldsymbol{\Lambda}+\frac{m_{s}(\mathbf{x})}{3} \boldsymbol{\theta}(\mathbf{x})+\boldsymbol{\phi}(\mathbf{x})$. If the difference of $\boldsymbol{\theta}$ for each color channel is considerably large $\left(\theta_{r} \neq \theta_{g} \neq \theta_{b}\right)$, the specular component $\left(\frac{m_{s}(\mathbf{x})}{3} \boldsymbol{\theta}(\mathbf{x})\right)$ will be different for each color channel (the specular components become non-scalar value), even if $\Gamma_{r}=\Gamma_{g}=\Gamma_{b}$. This 


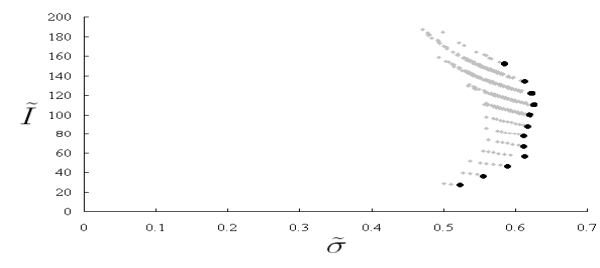

(a)

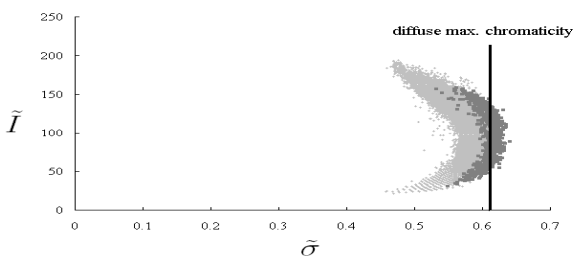

(b)

Figure 4: (a) Darker points represent diffuse point candidates (b) darker cloud represents diffuse point candidates for all curved lines of all values of $u$. The vertical line represents estimated actual diffuse maximum chromaticity.

makes specular points distribution not follow Equation (10), and as a result makes us fail to detect the specular curved lines distribution. However, since our purpose is to acquire diffuse maximum chromaticity, the failure to detect the specular curved lines can benefit us. In contrast, if $\theta_{r} \approx \theta_{g} \approx$ $\theta_{b}$ and $\Gamma_{r} \approx \Gamma_{g} \approx \Gamma_{b}$, using Equation (10) we could possibly identify the specular curved lines distribution when detecting diffuse curved lines, which consequently produces a potential problem in estimating actual diffuse maximum chromaticity. Fortunately, our technique to choose the diffuse point candidates from the lowest intensity of each curved line enables us to avoid this problem. The reason is, most of the lowest intensity of every specular curved line whose specular component is approximately scalar, also indicates the actual diffuse point. In cases where there are specular curved lines that have no diffuse points, we deem them to be outliers whose number is usually smaller than the number of diffuse point candidates. Figure 4.a shows the diffuse points that correspond to diffuse pixel candidates for a single value of $u$. In this case, the specular points distribution follows Equation (10). Figure 4.b shows the diffuse candidates from all curved lines of all groups of $u$.

Finally, to obtain a unique value of maximum chromaticity from diffuse point candidates, we simply use histogram analysis. We count the number of diffuse point candidates for each value of maximum chromaticity, and choose the largest count as the diffuse maximum chromaticity. Figure 4.b shows a vertical line that illustrates the candidates in maximum chromaticity-intensity space and its single estimated diffuse maximum chromaticity. Note that the noise characteristics explained in this section can be found if the camera output is linear to incoming light intensity and $\phi_{r} \approx \phi_{g} \approx \phi_{b}$. In addition, in case a camera does not have the second type of camera noise, the diffuse maximum chromaticity identification becomes more straightforward as the diffuse points will form a vertical line in maximum chromaticity intensity space.

\subsection{Non-White Illumination and Normalization}

In the real world, finding a pure-white specular component is almost impossible. Most light sources are not wavelength-independent. Moreover, even if the light source is pure white, because of sensor sensitivity and camera noise, the value of the specular component will be different for each color 
channel. With this condition, for identifying diffuse points candidates, non-white specular components benefit us, since they make diffuse point candidates identification more robust. As explained in the previous section (3.2), illumination color and sensor sensitivity do not affect the second type of noise $(\phi)$. Thus, we can still identify diffuse maximum chromaticity whatever the illumination color and sensor sensitivity function. In other words, the identification of diffuse candidates under non-white and white illumination is exactly the same.

Reflection components separation using specular-to-diffuse mechanism requires that the specular component be pure-white or identical for all color channels. Consequently, we have to make the specular component become a scalar value (normalization process). We propose a simple normalization technique without using color basis functions, namely, dividing each pixel's RGB with illumination chromaticity. To estimate illumination chromaticity, we can use color constancy algorithms that can handle uniformly colored surfaces such as [14, 2].

Having estimated the illumination chromaticity $\left(\Gamma^{e s t}\right)$, the normalized image intensity becomes:

$$
\frac{\mathbf{I}(\mathbf{x})}{\boldsymbol{\Gamma}^{e s t}}=\frac{m_{d}(\mathbf{x}) \boldsymbol{\Lambda} \boldsymbol{\theta}(\mathbf{x})+\boldsymbol{\phi}(\mathbf{x})}{\boldsymbol{\Gamma}^{e s t}}+\frac{m_{s}(\mathbf{x}) \boldsymbol{\Gamma} \boldsymbol{\theta}(\mathbf{x})}{\boldsymbol{\Gamma}^{e s t}}
$$

By assuming $\frac{\boldsymbol{\Gamma} \boldsymbol{\theta}(\mathbf{x})}{\boldsymbol{\Gamma}^{e s t}}=\{t, t, t\}$, where $t$ is a scalar value, we can obtain pure-white specular reflection component. This assumption is reasonable, since the estimation value of $\Gamma^{e s t}$ either using a color constancy algorithm or white reference (taken by the same camera) also affected by the same noise.

Having normalized both input image pixels and diffuse pixel candidates, and then computing the normalized diffuse maximum chromaticity, we can directly separate normalized diffuse and specular components using the specular-to-diffuse mechanism. Finally, in order to obtain the actual diffuse and specular components, we have to renormalize the separated reflection component by multiplying them with $\Gamma^{e s t}$ (the illumination chromaticity).

\section{Experimental Results}

In this section we first briefly describe the implementation of the proposed method, and then present several experimental results on real input images.

Implementation Figure 5 shows the flowchart of our method. Given an input image of uniformly colored surfaces, we first group the pixels of the image based on their color ratio $(u)$ values. Then, for every group of $u$, we identify the diffuse point candidates, which implies identifying the diffuse pixel candidates. Using estimated illumination chromaticity, we normalize all diffuse pixels candidates as well as the input image. Based on the normalized diffuse pixel candidates, using histogram analysis we calculate a unique value of normalized diffuse maximum chromaticity. By knowing the normalized diffuse chromaticity, we separate the normalized input image by using the specular-to-diffuse 


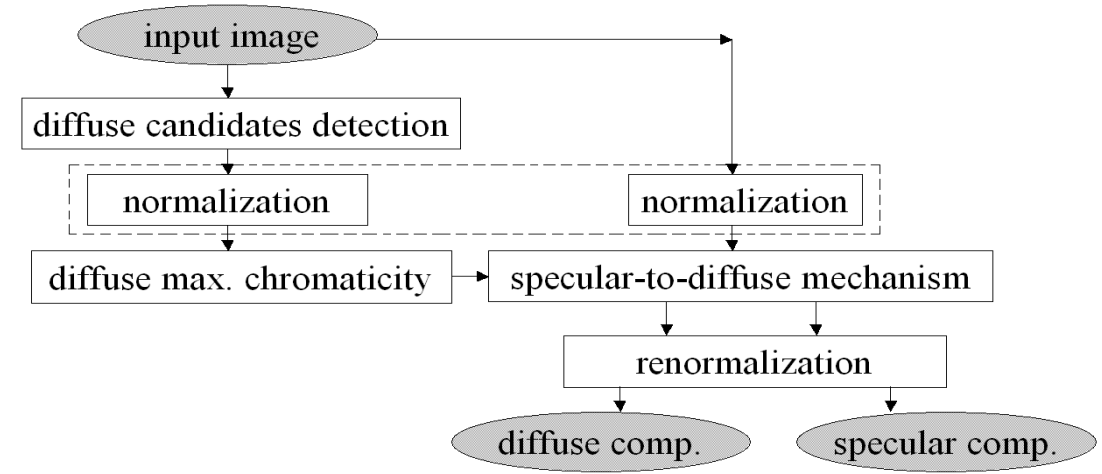

Figure 5: Flowchart of the proposed method

mechanism, producing normalized diffuse and specular components. Finally, to obtain the actual components, we multiply both normalized components by the estimated illumination chromaticity. The method can be implemented to handle multicolored surfaces by using color-ratio or hue-based color segmentation; both color ratio (Equation 13) and hue value will be independent from specularity if the specular reflection component is pure-white.

We have conducted several experiments on real images captured by using three different CCD cameras: SONY DXC-9000 (a progressive 3 CCD digital camera), Victor KY-F70 (a progressive 3 CCD digital camera), and SONY XC-55 (a monochrome digital camera with external color filters). To estimate illumination chromaticity, we used an existing illumination chromaticity estimation algorithm [14]. In our experiments, we used convex objects to avoid interreflection.

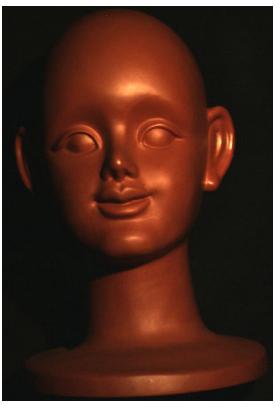

(a)

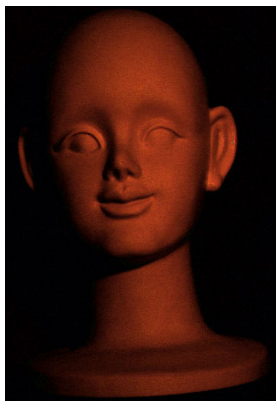

(b)

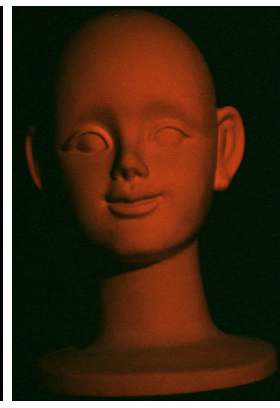

(c)

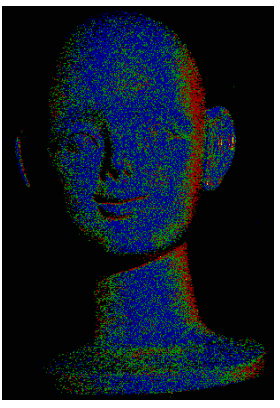

(d)

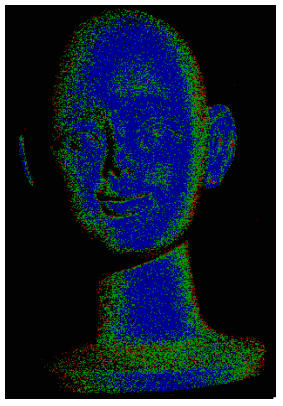

(e)

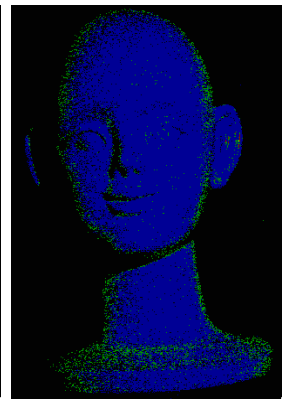

(f)

Figure 6: (a) Input image (b) estimated diffuse reflection component (c) diffuse reflection component by using polarizing filters (d) error in red channel (e) error in green channel (f) error in blue channel.

Evaluation We evaluated the estimation results by comparing with the results of using two polarizing filters. We placed one of the two filters in front of camera and another filter in front of the light source. Theoretically, if we change the polarization angle of one of the two filters into a certain angle that is opposite to another filter, we will obtain diffuse only reflection. Figure 6.a, b and c show the input image, the diffuse reflection component estimated using our method and the diffuse reflec- 


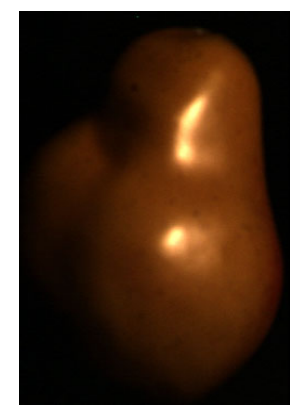

(a)

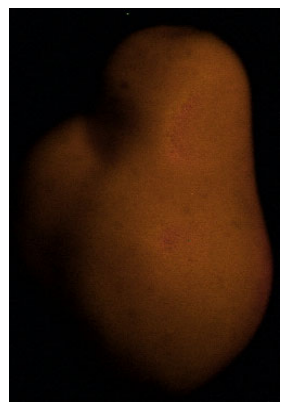

(b)

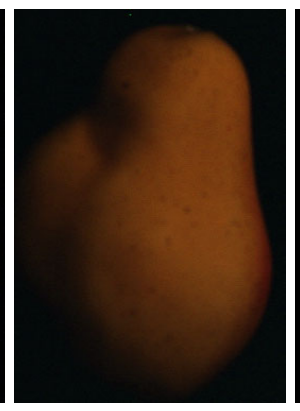

(c)

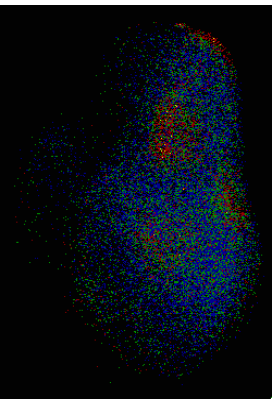

(d)

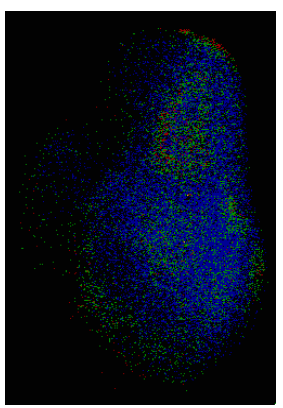

(e)

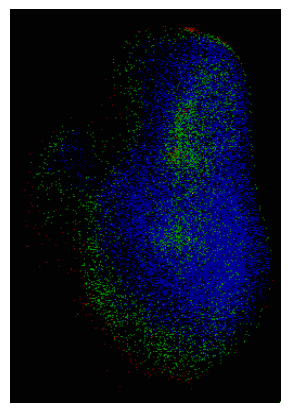

(f)

Figure 7: (a) Input image (b) estimated diffuse reflection component (c) diffuse reflection component by using polarizing filters (d) error in red channel (e) error in green channel (f) error in blue channel.

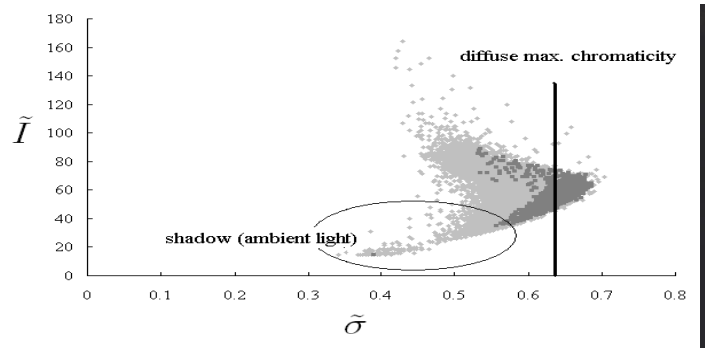

(a)

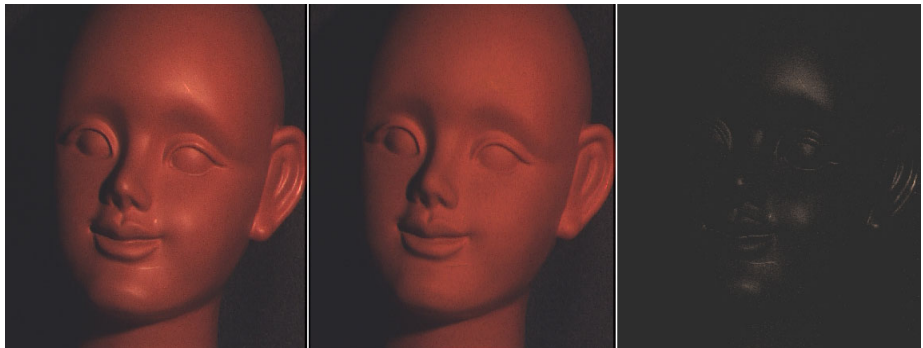

(c) (d)

Figure 8: (a) Diffuse maximum chromaticity estimation for an image taken by Victor KY-F70 (b) Input image (c) diffuse reflection component (d) specular reflection component.

tion component obtained using the two polarizing filters, respectively. Figure 6.c, $d$ and e shows the difference of image intensity values in red, blue and green color channel. Blue pixels in the figures represent $0 \sim 5$ pixel intensity difference. Green pixels represent $6 \sim 10$ pixel intensity difference, while red pixels represent $11-20$ pixel intensity difference. Green pixels occurred dominantly in the comparison, particularly for red channel. This is due to various factors, such as, inaccurate illumination chromaticity estimation, which implies inaccurate grouping of $u$ and inaccurate separation using specular-to-diffuse mechanism; the assumption that $\phi_{r}=\phi_{g}=\phi_{b}$ does not hold precisely; and, the second type of noise (dark noise, $\phi$ ) that also occurs in the results of using polarizing filters. However, despite these factors, the estimation results are considerably accurate, since the maximum value of second type of noise of the camera (Sony DXC-9000) is around 10. Figure 7 shows another separation result using different object. Note that, in this evaluation we do not evaluate pixels whose image intensity is below camera dark.

To examine the robustness of the proposed methods, we also conducted several experiments using three different cameras. Figure 8.a shows the estimation of actual diffuse maximum chromaticity for Victor KY-F70. Although some points that, due to ambient light in shadow regions, produce uncharacterized distribution, the diffuse chromaticity was still correctly obtained. The separation result using this camera is shown in Figure $8 \mathrm{c}-\mathrm{d}$. Figure 9 shows the separation results using SONY XC-55, 


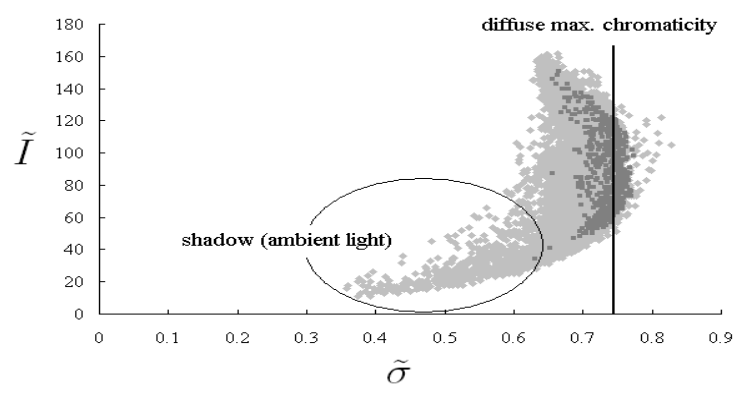

(a)

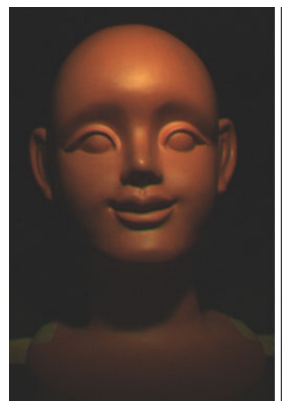

(b)

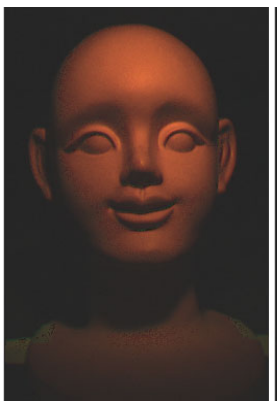

(c)

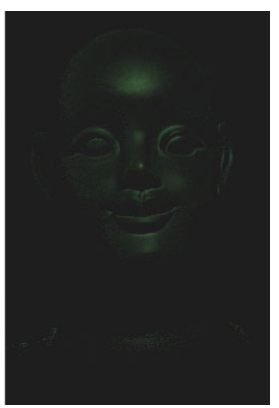

(d)

Figure 9: (a) Diffuse maximum chromaticity estimation for an image taken by SONY XC-55 (b) input image (c) diffuse reflection component (d) specular reflection component.
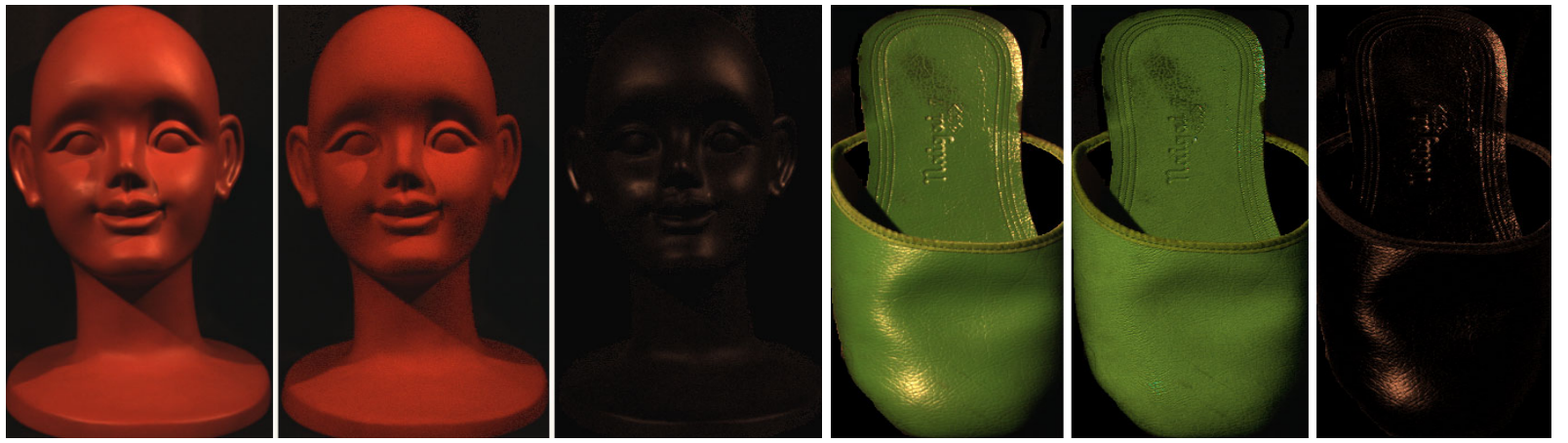

Figure 10: Reflection components separation for images captured by SONY DXC-9000

a monochrome camera with external color filters. Figure 10 show the results using Sony DXC-9000.

For more results, please visit our website: www.cvl.iis.u-tokyo.ac.jp/ robby/separation/results.html.

\section{Conclusion}

We have proposed a novel method to separate diffuse and specular reflection components. The main insight of our method is to analyze specular and diffuse pixels distribution in maximum chromaticityintensity space, in addition to analyzing noise for more robust and accurate results. To identify diffuse chromaticity and to separate reflection components, we introduced specular-to-diffuse mechanism. This mechanism is accurate in separating the reflection components when given only the diffuse chromaticity of the normalized image.

\section{Appendix}

Derivation of the correlation between illumination chromaticity and image chromaticity.

$$
\boldsymbol{\sigma}(\mathbf{x})=\frac{m_{d}(\mathbf{x}) \boldsymbol{\Lambda}+m_{s}(\mathbf{x}) \boldsymbol{\Gamma}}{m_{d}(\mathbf{x})\left[\Lambda_{r}+\Lambda_{g}+\Lambda_{b}\right]+m_{s}(\mathbf{x})\left[\Gamma_{r}+\Gamma_{g}+\Gamma_{b}\right]}
$$


For local (pixel based) operation the location $(\mathbf{x})$ can be removed. Then:

$$
m_{s}=m_{d} \frac{(\boldsymbol{\Lambda}-\boldsymbol{\sigma})}{(\boldsymbol{\sigma}-\boldsymbol{\Gamma})}
$$

Substituting $m_{s}$ in the definition of $\mathbf{I}$ with $m_{s}$ in the last equation:

$$
\mathbf{I}=m_{d}(\boldsymbol{\Lambda}-\boldsymbol{\Gamma})\left(\frac{\boldsymbol{\sigma}}{\boldsymbol{\sigma}-\boldsymbol{\Gamma}}\right)
$$

Since $\Gamma_{r}+\Gamma_{g}+\Gamma_{b}=1$, and under the assumption of achromatic illumination, $\Gamma_{r}=\Gamma_{g}=\Gamma_{b}$ then $\boldsymbol{\Gamma}=\{1 / 3,1 / 3,1 / 3\}$.

\section{References}

[1] R. Bajscy, S.W. Lee, and A. Leonardis. Detection of diffuse and specular interface reflections by color image segmentation. International Journal of Computer Vision, 17(3):249-272, 1996.

[2] G.D. Finlayson and G. Schaefer. Solving for color constancy using a constrained dichromatic reflection model. International Journal of Computer Vision, 42(3):127-144, 2001.

[3] G. Healey and R. Kondepudy. Radiometric ccd camera calibration and noise estimation. IEEE Trans. on Pattern Analysis and Machine Intelligence, 16(3):267-276, 1994.

[4] G.J. Klinker, S.A. Shafer, and T. Kanade. The measurement of highlights in color images. International Journal of Computer Vision, 2:7-32, 1990.

[5] H.C. Lee. Method for computing the scene-illuminant from specular highlights. Journal of Optics Society of America A., 3(10):1694-1699, 1986.

[6] H.C. Lee, E.J. Breneman, and C.P.Schulte. Modeling light reflection for computer color vision. IEEE Trans. on Pattern Analysis and Machine Intelligence, 12:402-409, 1990.

[7] S.W. Lee and R. Bajcsy. Detection of specularity using color and multiple views. Image and Vision Computing, 10:643-653, 1990.

[8] A. Leonardis, A. Gupta, and R. Bajcsy. Segmentation as the search for the best description in terms of primitives. in proceeding of IEEE International Conference on Computer Vision, pages 121-125, 1990.

[9] S. Lin, Y. Li, S. B. Kang, X. Tong, and H.Y. Shum. Diffuse-specular separation and depth recovery from image sequences. In in proceeding of European Conference on Computer Vision, pages 210-224, 2002.

[10] S. Lin and H.Y. Shum. Separation of diffuse and specular reflection in color images. In in proceeding of IEEE Conference on Computer Vision and Pattern Recognition, 2001.

[11] S.K. Nayar, X.S. Fang, and T. Boult. Separation of reflection components using color and polarization. International Journal of Computer Vision, 21(3), 1996.

[12] Y. Sato and K. Ikeuchi. Temporal-color space analysis of reflection. Journal of Optics Society of America A., 11(11):2990-3002, 1994.

[13] S. Shafer. Using color to separate reflection components. Color Research and Applications, 10:43-51, 1985.

[14] R. T. Tan, K. Nishino, and K. Ikeuchi. Illumination chromaticity estimation using inverse-intensity chromaticity space. in proceeding of IEEE Conference on Computer Vision and Pattern Recognition, pages 673-680, 2003.

[15] L.B. Wolff and T. Boult. Constraining object features using polarization reflectance model. IEEE Trans. on Pattern Analysis and Machine Intelligence, 13(7):635-657, 1991. 